\title{
Method of the Year 2017: Organoids
}

\author{
The ability to prod stem cells into three-dimensional tissue models makes for a powerful way \\ to study human biology. But these exciting tools are still works in progress.
}

Life happens in (at least) three dimensions. Studying its molecular and cellular basis in flat cells attached to hard plastic, revealing though this is about some aspects of biology, will only take us so far. But scientists have recently added a shiny new tool to their belts: three-dimensional multicellular stem-cell-derived constructs that mimic in vivo tissue, or organoids. For their fascinating potential as tools to probe human biology and disease, we have chosen organoids as Method of the Year 2017.

Organoids are a refurbished rather than an entirely new tool; three-dimensional culture of cells and organ fragments is, after all, an old discipline. But it's only recently that knowledge of mammalian development and tissue homeostasis on the one hand, and of extracellular matrix biology on the other, have coalesced with growing experience in stem cell culture to channel the awesome self-organizing properties of stem cells and their derivatives into realistic in vitro models of human tissue. Organoid models have now been grown for many organs: brain, liver, kidney, breast, retina, and organs of the gastrointestinal tract, among others.

The potential applications of these constructs are equally varied; a News Feature (p19) surveys the possibilities. Organoids are obvious models for prenatal development and tissue maintenance, otherwise intractable processes to study in human. They provide a source of human tissue to study any number of biological processes; recent work reports the first observations of interneuron migration within living human tissue, for example. Organoids are being used to model disease-from infectious disease like Zika virus infection to single gene disorders like cystic fibrosis and complex conditions like cancer. More generally, since the starting stem cells can be edited to carry a genetic variant or mutation of interest, or can be derived from many genetic backgrounds, these could be powerful models for examining the phenotypic consequence of genotype. They have even been applied to evolutionary questions, permitting ambitious comparisons between human and ape tissue. A Commentary (p27) discusses the promise and also the challenges of using brain organoids derived from pluripotent stem cells to model this most complex of organs.

Perhaps one of the most powerful aspects of organoids as research tools is just how well they synergize with other cutting-edge methods. Imaging, for example, has been advancing tremendously within the past decade, particularly in its ability to image deeper into tissue in ways both fast enough to capture living biology and gentle enough not to damage the sample much. A second Commentary (p24) discusses how imaging of adult stem cell-derived organoids-in fixed or living tissue, at small scale or in high throughput, in vitro or upon reintroduction into the body-is advancing our understanding of human biology, especially in cancer.

Single-cell transcriptional profiling is another exploding area of methods development that is being put to good use on these samples, whether to characterize organoids that result from different culture methods, to trace lineage, or to read out the consequence of a perturbation. These methods yield a finer-grained picture than bulk approaches of both organoid cellular composition and of how closely the cells match their in vivo counterparts.

Organoids can contain an impressive range of cell types-kidney organoids, for example, have been reported to have at least ten, with genuinely complex structure. But, as is indeed to be expected from constructs generated in vitro, organoids remain imperfect models in terms of cell-type representation, structure, and mature function. These models will evolve; they will need to be continually evaluated and any biological interpretations tempered by an assessment of their limitations. Clearly, as well, experiments in animal models cannot be abandoned, and data on human fetal tissue will continue to be needed as a benchmark. As a consequence of their complexity and their generation via self-organization, these preparations are also prone to variability (discussed in a Primer on $\mathrm{p} 23$ ). Both technical and biological variation must be taken into account for organoids to reach their full utility.

Our special feature on these tools (p19-33; this includes also a section on methods to watch in coming years) is not intended to be comprehensive about the applications, the history, or the challenges of this nascent technology. We do not cover several related, but more peripheral areas; for instance, 'gastruloids', engineered embryonic stem cell colonies that display early lineage specification, or 'organ-on-a-chip' approaches that integrate microfluidic perfusion with three-dimensional culture. Our hope is to give our readers a flavor of the field and convey some of the exciting possibilities of human organoid culture.

We wish our readers a happy 2018 ! 